\title{
Promyelocytic sarcoma presenting with spinal cord compression and treated successfully with surgical debulking and the PETHEMA regimen for acute promyelocytic leukemia
}

\author{
Dennis Cornfield ${ }^{1}$, Shereen Gheith ${ }^{1}$, Lloyd Barron ${ }^{2}$ \\ 1. Hematopathology section, Dept. of Pathology, Health Network Laboratories/Lehigh Valley Health Network, Allentown, \\ Pennsylvania, United States. 2. Hematology/Medical Oncology section, Dept. of Medicine, Lehigh Valley Health Network, \\ Allentown, Pennsylvania, United States.
}

Correspondence: Shereen Gheith, M.D., PhD. Address: Dept. of Pathology, Lehigh Valley Health Network, 1200 S. Cedar Crest Blvd, Allentown, PA 18103, United States. E-mail: gheiths@comcast.net

Received: January 23, 2015

DOI : $10.5430 / \mathrm{crcp} . \mathrm{v} 2 \mathrm{n} 3 \mathrm{p} 12$
Accepted: March 2, 2015

URL: http://dx.doi.org/10.5430/crcp.v2n3p12

\section{Abstract}

A 52-year old woman presented with signs and symptoms of thoracic spinal cord compression caused by a spinal canal mass extending from the seventh to the ninth thoracic vertebra. Initial treatment consisted of high dose steroids and decompression laminectomy with subtotal resection of the abnormal mass. Sheets of immature myeloid cells which expressed the $\mathrm{t}(15 ; 17)(\mathrm{q} 22 ; \mathrm{q} 21)$ of acute promyelocytic leukemia (APL) by fluorescence in situ hybridization (FISH) were present, consistent with promyelocytic sarcoma. Bone marrow examination showed no evidence of acute leukemia. She was then placed on the PETHEMA (Programa para el Tratamiento de Hematopatias Malignas, a Spanish cooperative chemotherapy group) regimen for APL for 15 months. There has been no evidence of local recurrence or of systemic APL at the 4.5-year mark, the longest follow-up of isolated promyelocytic sarcoma in the medical literature.

\section{Key words}

Promyelocytic sarcoma, Leukemia, Cord compression, Extramedullary, Acute promyelocytic leukemia

\section{I ntroduction}

Myeloid sarcoma (MS) is an uncommon but well-described lesion consisting of an extramedullary mass of immature myeloid cells. Every subtype of acute myeloid leukemia has an MS counterpart. Promyelocytic sarcoma is an extremely rare form of MS corresponding to the malignant promyelocytes of acute promyelocytic leukemia (APL). When present, it most often occurs as a manifestation of relapsed APL or at the time of diagnosis of systemic APL ${ }^{[1,2]}$. This report describes a patient with an isolated promyelocytic sarcoma causing spinal cord compression and treated successfully with decompression laminectomy followed by the PETHEMA chemotherapy regimen for APL ${ }^{[3]}$. There has been no morphologic or molecular evidence of local recurrence or of systemic APL, either at diagnosis or in the ensuing 4.5 years. 


\section{Case presentation}

A 52-year old woman presented with signs and symptoms of thoracic spinal cord compression. Physical examination revealed no skin lesions or organomegaly. Complete blood count was entirely normal, with hemoglobin 13.6 g/dl, WBC $7,000 / \mu l$ with a normal differential (no blasts), platelet count 346,000/ $\mu$ l. Serum chemistry profile showed creatinine $0.8 \mathrm{mg} / \mathrm{dl}$ and albumin $4.7 \mathrm{~g} / \mathrm{dl}$. There was no evidence of a coagulopathy. Past medical history included papillary carcinoma of the thyroid six years earlier, treated with thyroidectomy and radioactive iodine. Magnetic resonance imaging (MRI) performed during the present admission demonstrated a mass lesion occupying the left side of the spinal canal and extending from the level of the seventh to the ninth thoracic vertebral bodies (see Figure 1). Emergency treatment consisted of high-dose dexamethasone and decompression laminectomy with partial resection of the epidural mass. Hematoxylin and eosin (H\&E)-stained sections of lesional tissue demonstrated sheets of immature-appearing mononuclear cells with eccentric nuclei and finely granular pink cytoplasm (see Figure 2). Fine needle aspirate (FNA) of the lesion consisted of mostly peripheral blood. A single cell with prominent Auer rods was identified, consistent with a malignant non-microgranular promyelocyte.

Figure 1. T2-weighted MRI shows a low signal intensity spinal mass extending from T7 to T9 (arrow)

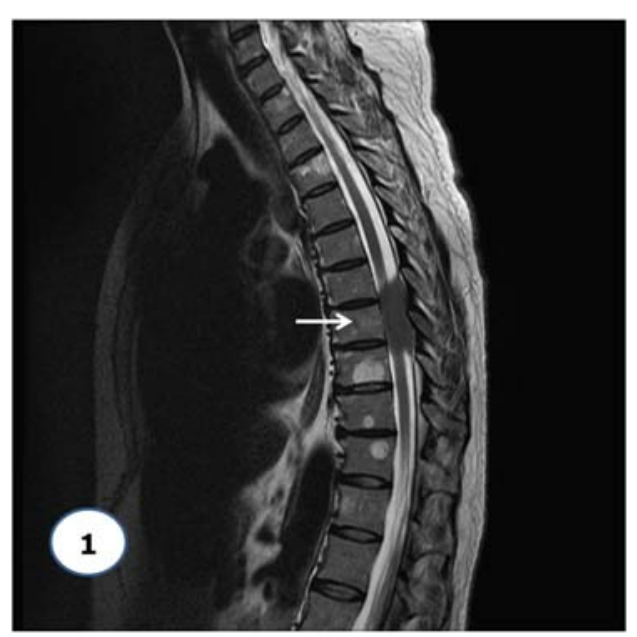

Figure 2. H\&E-stained section of the paraspinal mass shows sheets of mononuclear cells with pink-reddish cytoplasm, consistent with promyelocytes (H\&E stain, original magnification $\times 100$ ). A malignant promyelocyte with numerous Auer rods is demonstrated on the FNA (Wright-Giemsa stain, original magnification $\times 1,000)$.

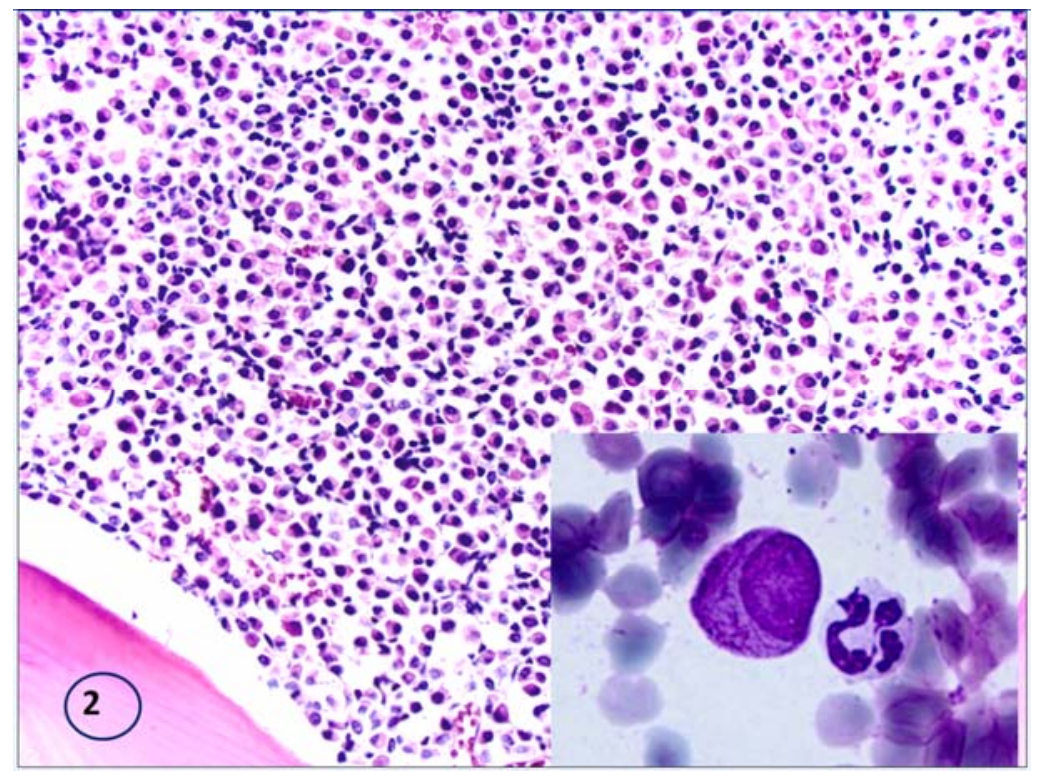

By immunohistochemistry, virtually $100 \%$ of the mononuclear cells were brightly positive for MPO (see Figure 3a), lysozyme, CD68 and CD33 and were negative for a large panel of hematopoietic and non-hematopoietic antibodies: CD34, CD45, CD117, CD20, CD43, PAX-5, CD3, CD4, CD8, CD138, kappa and lambda light chain, CD1a, CD163, 
CD79a, TdT, CD30, ALK-1, CD5, CD7, CD2, TTF-1, thyroglobulin, CAM5.2, AE1/AE3, HMB45 and MART-1. Fluorescence in situ hybridization (FISH) analysis of lesional tissue showed $t(15 ; 17)(q 22 ; q 21)$ gene rearrangement in 88.5\% of interphase nuclei (see Figure 3b), establishing a diagnosis of promyelocytic sarcoma. Iliac crest bone marrow sampling showed active trilineage hematopoiesis with no morphologic evidence of APL. Cytogenetic analysis of the bone marrow aspirate showed a normal (46, XX) chromosome karyotype. The patient was empirically started on the PETHEMA regimen for APL, with dose modifications as appropriate. This regimen consists of an induction phase using intravenous (iv) idarubicin and oral (po) all-trans retinoic acid (ATRA); a consolidation phase using po ATRA plus iv idarubicin alternating with mitoxantrone for 3 cycles; and a maintenance phase using po ATRA, methotrexate, and 6-mercaptopurine for two years ${ }^{[3]}$. The patient required two hospitalizations for neutropenic fever during the first two months of treatment. She improved each time on parenteral antibiotics and intravenous fluids. Because of the development of pulmonary edema and pleural effusions during the first hospitalization, the ATRA (differentiation) syndrome ${ }^{[4]}$ was suspected and was treated with dexamethasone, furosemide, and temporary withdrawal of ATRA. During the second hospitalization for neutropenic fever, Pseudomonas putida was identified on 1 blood culture. Once she was stable after discharge, she continued on the PETHEMA regimen and experienced no significant therapy-related complications. There has been a gradual, sustained improvement in her neurologic status. Periodic radiologic studies, bone marrow examination, and molecular studies for the t(15;17)(q22;q21) on peripheral blood and bone marrow have shown no evidence of recurrent promyelocytic sarcoma or of systemic APL. RT-PCR for $t(15 ; 17)(q 22$; q21), performed on a bone marrow sample obtained after six months of treatment and on peripheral blood at the four year mark, was negative on both occasions. Because of the chemotherapy-related cytopenias noted above, and in view of the lack of guidelines for duration of systemic treatment for promyelocytic sarcoma, methotrexate and 6-mercaptopurine were discontinued after 13 months; all-trans retinoic acid (ATRA) was stopped two months later. She remains free of disease at the 4.5-year mark post initiation of treatment.

Figure 3. Immunohistochemistry shows $\mathrm{MPO}(+)$ promyelocytes (original magnification $\times 200)(a)$. On FISH analysis, $P M L / R A R A$ gene rearrangement is identified by 2 yellow (fusion), 1 red (PML) and 1 green $(R A R A)$ signals (b).

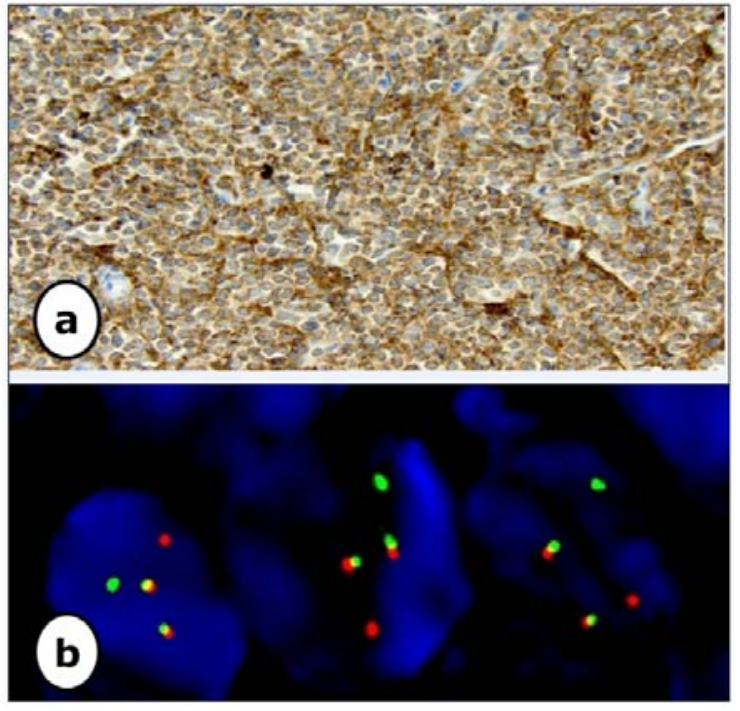

\section{Discussion}

Promyelocytic sarcoma occurring in the setting of APL is an uncommon event. The majority of cases reported in the literature have been found either concurrent with the diagnosis of APL or especially at relapse of APL. In 1996, Wiernik et al. ${ }^{[1]}$ reported on two cases of extramedullary promyelocytic leukemia (promyelocytic sarcoma) presenting as relapsed disease and reviewed 24 additional cases of extramedullary APL in the medical literature. Of the 26 total cases, two were diagnosed shortly before documented bone marrow involvement by APL, five cases simultaneous with bone marrow involvement, and 19 cases after the initial diagnosis of APL, including 11 patients who were in complete remission. The skin was involved in 15 of the cases of extramedullary APL, followed by central nervous system (CNS, five patients), 
mediastinum (three patients) and gingiva (three patients). Because extramedullary APL occurred in 19 patients who had received ATRA as part of their anti-leukemia regimen, the suggestion was made that ATRA might possibly play a role in the development of extramedullary APL. Two years later, a report from Italy ${ }^{[2]}$ documented the occurrence of extramedullary APL in seven patients out of 120 adults with APL referred to 2 institutions over a nine year period. The authors noted that the medical literature contained 37 well-documented cases of extramedullary APL. Six of their own seven cases were diagnosed at the time of relapsed APL; 1 case occurred simultaneous with the initial diagnosis of APL. The sites of disease were skin (5 cases), CNS (1 case), and lymph node ( 1 case). In the six patients who relapsed with extramedullary APL, 2 (of 61 patients) had received chemotherapy plus ATRA and 4 (of 59 patients) had received chemotherapy alone, leading the authors to conclude that ATRA does not appear to predispose to the development of extramedullary APL any more than other types of treatment for APL. De Botton et al. analyzed 169 relapses of 740 patients with APL treated in 3 multicenter trials ${ }^{[5]}$. Ten of the 169 relapses were extramedullary, involving the CNS (9 cases) and skin (1 case). By multivariate analysis, an elevated white blood cell count at diagnosis was found to be a significant risk factor for extramedullary relapse. The variability in sites of extramedullary relapse of APL was underscored by Tirado et al, who described multiple relapses in numerous anatomic sites, including right atrium of the heart, left scapula, thoracic vertebra, and supraclavicular mass, in a 30-year old with APL with cryptic PML-RARA fusions ${ }^{[6]}$.

When considering promyelocytic sarcoma specifically involving the spine, we are aware of only 5 cases in the medical literature. One patient had thoracic epidural compression presenting 10 months before a diagnosis of systemic APL ${ }^{[7]}$; 2 had epidural or spinal masses at the time of diagnosis of APL ${ }^{[8,9]}$; and 2 had a thoracic spinal ${ }^{[10]}$ or paraspinal ${ }^{[11]}$ mass as the only evidence of relapsed APL. These patients were treated with combinations of surgery, radiotherapy, and chemotherapy, including ATRA, and achieved complete responses for variable periods of time.

The occurrence of isolated promyelocytic sarcoma in a completely non-leukemic setting is exceedingly rare. In some of the cases reported in the pre-molecular era, the diagnosis was retrospective and based on tissue obtained at relapse ${ }^{[7,12,13]}$. Two recently-reported cases, involving a sternal mass ${ }^{[14]}$ and a sacral epidural mass ${ }^{[15]}$, were diagnosed based on molecular demonstration of the PML-RARA fusion gene in lesional tissue. The longest follow-up was 13 months ${ }^{[15]}$. In our case, promyelocytic sarcoma was confirmed by FISH demonstration of $t(15 ; 17)(q 22 ; q 21)$ on the epidural mass. The 4.5-year follow-up is the longest in the medical literature.

The optimal treatment of extramedullary myeloid sarcomas is unknown. In a 1995 retrospective review of all published cases of extramedullary nonlymphocytic leukemia since $1965^{[16]}$, the authors found that all but $3 \%$ of cases progressed to systemic acute leukemia in the absence of treatment with chemotherapy, as opposed to $66 \%$ of patients who did not develop acute leukemia after treatment with chemotherapy. It is now generally felt that chemotherapy appropriate for the specific type of myeloid sarcoma present is the preferred approach to forestall or prevent the emergence of systemic acute leukemia. The PETHEMA regimen was used in the present case because of its documented success in APL. Exactly what factor(s) is responsible for this patient's prolonged disease-free survival, with no evidence of local recurrence or progression to APL, is unclear. The answer may involve a combination of low total body burden of disease after surgical debulking; a drug regimen highly effective against APL; use of maintenance therapy after achievement of a molecular remission; and sheer good fortune.

\section{Conclusion}

To the authors' knowledge, this case represents the longest (4.5 years) follow-up of isolated promyelocytic sarcoma in the medical literature. Although the experience of a single patient does not warrant a generalization, it would appear that the PETHEMA regimen, as used in the present case, may be an effective approach to treatment of this rare entity. 


\section{Conflict of interests}

The authors declare that they have no conflict of interests.

\section{References}

[1] Wiernik PH, De Bellis R, Muxi P, et al. Extramedullary acute promyelocytic leukemia. Cancer. 1996; 78: $2510-2514$. http://dx.doi.org/10.1002/(SICI)1097-0142(19961215)78:12<2510::AID-CNCR10>3.0.CO;2-Z

[2] Liso V, Specchia G, Pogliani EM, et al. Extramedullary involvement in patients with acute promyelocytic leukemia: a report of seven cases. Cancer. 1998; 83: 1522-1528. http://dx.doi.org/10.1002/(SICI)1097-0142(19981015)83:8<1522::AID-CNCR6>3.0.CO;2-4

[3] Sanz MA, Vellenga E, Rayon C, et al. All-trans retinoic acid and anthracycline monochemotherapy for the treatment of elderly patients with acute promyelocytic leukemia. Blood. 2004; 104: 3490-3493. PMid: 15292063. http://dx.doi.org/10.1182/blood-2004-04-1642

[4] Montesinos P, Bergua JM, Vellenga E, et al. Differentiation syndrome in patients with acute promyelocytic leukemia treated with all-trans retinoic acid and anthracycline chemotherapy: characteristics, outcome, and prognostic factors. Blood. 2009; 113: 775-783. PMid: 18945964. http://dx.doi.org/10.1182/blood-2008-07-168617

[5] de Botton S, Sanz MA, Chevret S, et al. Extramedullary relapse in acute promyelocytic leukemia treated with all-trans retinoic acid and chemotherapy. Leukemia. 2006; 20: 35-41. PMid: 16307026. http://dx.doi.org/10.1038/sj.leu.2404006

[6] Tirado CA, Chen W, Valdez F, et al. Unusual presentation of myeloid sarcoma in a case of acute promyelocytic leukemia with a cryptic PML-RARA rearrangement involving multiple sites including the atrium. Cancer Genet Cytogenet. 2010; 1: 47-53. PMid: 20513534. http://dx.doi.org/10.1016/j.cancergencyto.2010.03.016

[7] Savranlar A, Ustündag Y, Ozer T, et al. A thoracic-epidural granulocytic sarcoma case that was diagnosed preceding the onset of and that recurred co-incidental to acute promyelocytic leukemia, which developed after surgical treatment. Acta Med Okayama. 2004; 5: 251-254.

[8] Bittencourt H, Teixeira Junior AL, Glória AB, et al. Acute promyelocytic leukemia presenting as an extradural mass. Rev Bras Hematol Hemoter. 2011; 6: 478-480. PMid: 23049367. http://dx.doi.org/10.5581/1516-8484.20110126

[9] Kyaw TZ, Maniam JA, Bee PC, et al. Myeloid sarcoma: an unusual presentation of acute promyelocytic leukemia causing spinal cord compression. Turk J Haematol. 2012; 3: 278-282. PMid: 24744674. http://dx.doi.org/10.5505/tjh.2012.94809

[10] Pacilli L, Lo Coco F, Ramadan SM, et al. Promyelocytic sarcoma of the spine: a case report and review of the literature. Adv Hematol. 2010; ID: 137608. 7 pages.

[11] Tsimberidou AM, Estey E, Whitman GJ, et al. Extramedullary relapse in a patient with acute promyelocytic leukemia: successful treatment with arsenic trioxide, all-trans retinoic acid and gemtuzumab ozogamicin therapies. Leuk Res. 2004; 9: 991-994. PMid: 15234578. http://dx.doi.org/10.1016/j.leukres.2004.01.004

[12] Kubonishi I, Ohtsuki Y, Machida K, et al. Granulocytic sarcoma presenting as a mediastinal tumor. Report of a case and cytological and cytochemical studies of tumor cells in vivo and in vitro. Am J Clin Pathol. 1984; 82: 730-734. PMid: 6594928.

[13] Zuiable A, Aboud H, Nandi A, et al. Extramedullary disease initially without bone marrow involvement in acute promyelocytic leukaemia. Clin Lab Haematol. 1989; 11: 288-289. PMid: 2591162. http://dx.doi.org/10.1111/j.1365-2257.1989.tb00223.x

[14] Thomas X, Chelghoum Y. Promyelocytic sarcoma of the sternum: a case report and review of the literature. Korean J Hematol. 2011; 46: 52-56. PMid: 21461306. http://dx.doi.org/10.5045/kjh.2011.46.1.52

[15] Fiegl M, Rieger C, Braess J, et al. Isolated epidural chloroma with translocation $\mathrm{t}(15 ; 17)$ successfully treated with chemotherapy and all-trans-retinoic acid. Br J Haematol. 2003; 122: 688-689. PMid: 12899728. http://dx.doi.org/10.1046/j.1365-2141.2003.04490.x

[16] Byrd JC, Edenfield WJ, Shields DJ, et al. Extramedullary myeloid cell tumors in acute nonlymphocytic leukemia: a clinical review. J Clin Oncol. 1995; 13: 1800-1816. PMid: 7602369. 